\title{
Teaching Quality Evaluation Model under the Background of Class Stratified System
}

\author{
Yunxia Zhang ${ }^{1, \mathrm{a}^{*}}$, Ping Liu, Jinfeng Lv ${ }^{3, c}$, Jingzhong $\mathrm{Li}^{4, \mathrm{~d}}$ \\ ${ }^{1}$ Hebei Normal University of Science \& Technology Qinhuangdao, Hebei Province, China \\ ${ }^{2}$ Qin Huangdao No.1 Senior High School Qinhuangdao, Hebei Province, China \\ ${ }^{3}$ Hebei Normal University of Science \& Technology Qinhuangdao, Hebei Province, China \\ ${ }^{4}$ Qin Huangdao No.1 Senior High School Qinhuangdao, Hebei Province, China
}

\begin{abstract}
Teaching quality evaluation of teacher is an important link in education and teaching activities, and an important index to measure the teaching level of teachers. It affects the level of a school and restricts the learning effect of students. The reform of college entrance examination system has brought new challenges to teachers' teaching quality evaluation. The traditional evaluation method based on absolute average score and standard deviation has been unable to meet the needs of the new teaching model. In this paper, by using "CEACV method", it will based on students' achievement and theory of value-added evaluation, calculate the average score and the coefficient of variation of several examination results in each class and comprehensively consider the mean of average score and coefficient of variation increments from students' class. Then teaching quality evaluation of teachers will be realized, with the mean of increments are weighted and sorted respectively, and get the final ranking with value-added method which based on the mean ranking of average score and coefficient of variation increments. The results show that "CEACV method" is scientific and effective, and can evaluate the teaching quality fairly and justly.
\end{abstract}

\section{Introduction}

In 2014, the implementation opinions on the Academic Level Examination for ordinary senior high schools were issued by the Ministry of Education of the People's Republic of China, which clearly pointed out that the reform of the college entrance examination and enrollment system should be promoted deeply. Impel the comprehensive implementation of class - based stratified teaching in China. Thus form a class-based stratified teaching model and a evaluation system of academic examination. In 2017, Zhejiang and Shanghai became the first batch of pilot reforms, and then the pilots have been expanded to many provinces and cities across the country quickly. The model of class-based stratified teaching is a great breakthrough, and make up for a lack of traditional administrative class in school. It will provide required and elective courses with different levels and different categories facing all students. They choose the courses that suit them and go to the appropriate classroom for studing, according to their own knowledge base, interest and demand in every subject under the guidance of teachers ${ }^{[1]}$. It fully respects students' right to choose, makes up for the inadequacy that students' differences cannot be taken into account in traditional administrative class, and promotes students to study with individuality ${ }^{[2]}$. Then some great challenges for educators are following. How to re-structure the evaluation system of off-class courses? How to control the learning state of students? How to evaluate the teaching effect of teachers? All these Have become the problems which are imminent, urgent need to face and solve.

Among the many domestic and foreign literatures on teaching quality evaluation of teacher ${ }^{[3-5]}$, there are very few literatures under the class-based stratified teaching mode. In 2016, Yingxiao Lv studied the evaluation strategy of teachers' teaching behavior against the background of the shift system, discussed the indirect evaluation based on learning results and the direct evaluation based on teaching process, and put forward the result-based and process-based suggestions for teachers' teaching evaluation ${ }^{[3]}$. In 2018, Kaijian Xiao put forward suggestions on the construction of the teaching evaluation system of Hainan high school history education under the class-based stratified teaching mode ${ }^{[2]}$. In this paper, it will based on students' achievement and theory of valueadded evaluation, calculate the average score and the coefficient of variation of several examination results in each class and comprehensively consider the mean of average score and coefficient of variation increments from Students' Class. Then teaching quality evaluation of teachers will be realized, with the mean of increments are weighted and sorted respectively, and get the final ranking of value-added method which based on the ranking by the mean of average score and coefficient of variation increments. 


\section{Evaluation method description}

Average score and standard deviation are both crucial indicators in the teaching quality evaluation, which are positively and negatively correlated with the class subject teaching quality respectively, no matter in the traditional teaching mode or class-based stratified teaching mode. The higher the average score is, the better the class scores are. The lower the average score is, the worse the class test scores are. The higher the standard deviation is, the higher the degree of discretization of the test scores, and the more dispersed the score distribution is. The lower the standard deviation is, the lower the degree of discretization and the more concentrated the grade distribution is. Class-based stratified teaching makes the students' achievement gap larger between different classes, and the categories become more obvious. The traditional longitudinal evaluation method according to the absolute average score and the standard deviation that based on a subject examination to measure students' achievement and teaching level of teachers has been unable to meet the needs of the new teaching mode. the meaning of longitudinal comparison based on average score and standard deviation among different levels and categories of class is lost. It is difficult to evaluate teaching quality and verify teachers' work performance.

This article improve the traditional method, according to the theory of value-added, which based on students' test scores for many times and the traditional evaluation method, with introducing the variation coefficient, it can eliminate the differences between students in different levels. And use the mode of horizontally to longitudinal insteading of the single longitudinal evaluation. Then the changes on performance of each class over a period of time can be evaluated comprehensively, through the average score and coefficient of variation increments. This method will be referred to as " comprehensive evaluation method based on the increments of average score and coefficient of variation ", shorthand for: "CEACV method".

Teaching quality evaluation for teachers of a certain grade is realized by " CEACV method" according to seven steps. The calculation of average score and standard deviation. The calculation of coefficient of variation. The calculation of the mean of average score increments. The calculation of the mean of coefficient of variation increments, the mean ranking of average score increments. The mean ranking of coefficient of variation increments, and the final ranking of value-added method.

\subsection{Calculate average score and standard deviation}

For a certain subject, the average score $A_{i j}, i=1,2, \cdots, m, \quad j=1,2, \cdots, n$ and the standard deviation $S_{i j}, i=1,2, \cdots, m, \quad j=1,2, \cdots, n$ of each class in each exam are calculated respectively by taking several test scores of students as the basic data (where $m$ represents the examination order, and $n$ represents the class serial number). Among them, the specific selection of tests to participate in the evaluation of teachers' teaching quality should be determined according to the actual examination situation of the school and their importance.

\subsection{Calculate coefficient of variation}

The coefficient of variation is determined by the average score $A_{i j}, i=1,2, \cdots, m, j=1,2, \cdots, n$ and standard deviation $S_{i j}, i=1,2, \cdots, m, j=1,2, \cdots, n$, and it will be got according to the formula (1) for each class in each exam, i.e

$$
V_{i j}=\frac{S_{i j}}{A_{i j}}, i=1,2, \cdots, m, j=1,2, \cdots, n .
$$

They cannot measure performance differences of discipline among each class directly by using average score and standard deviation, but the coefficient of variation processed by both them can successfully eliminate the differences on categories and levels of classes. It makes the scores of classes at different levels comparable, and provides a feasible path for teaching quality evaluation of teachers on class-based stratified teaching model. Subject coefficient of variation reflects the deviation degree of students' performance based on the average score of each unit within a class. It is an important indicator to measure the quality of class examination results, and has a negative correlation with the quality of class subject teaching, which doesn't restricted by levels and categories. The higher the coefficient of variation is, the higher the degree of discretization and the more dispersed the grade distribution is. The lower the coefficient of variation is, the lower the degree of discretization and the more concentrated the grade distribution is.

\subsection{Calculate the mean of average score increments}

The average scores of any two adjacent tests are subtracted, which means that the previous term is subtracted from the latter term, the result is called average score increment, denoted as,

$$
\begin{gathered}
U_{k j}, k=1,2, \cdots, m-1, j=1,2, \cdots, n \text {,i.e } \\
U_{k j}=A_{i+1, j}-A_{i j}, \\
k=i=1,2, \cdots, m-1, j=1,2, \cdots, n .
\end{gathered}
$$

The average score increments reflect the fluctuation of internal grade of class, and can grasp the change trend of class performance in time. By calculating the weighted mean of the average score increments for several tests, the mean of the average score increments can be obtained, i.e

$$
\bar{U}=\sum_{k=1}^{m-1} \lambda_{k} U_{k j}, j=1,2, \cdots, n .
$$
the

Where $\lambda_{k}, k=1,2, \cdots, m-1$ is the kth weight of 
average score increment, and the specific value can be determined according to the actual test situation.

\subsection{Calculate the mean of coefficient of variation increments}

The coefficient of variation of any two adjacent tests are subtracted, which means that the previous term is subtracted from the latter term, the result is called coefficient of variation increment, denoted as $\Delta_{k j}, k=1,2, \cdots, m-1, j=1,2, \cdots, n$,

i.e,

$$
\begin{gathered}
\Delta_{k j}=V_{i+1, j}-V_{i j}, \\
k=i=1,2, \cdots, m-1, j=1,2, \cdots, n .
\end{gathered}
$$

The coefficient of variation increments reflects the fluctuation of the degree of dispersion of grades within the class. and can grasp the distribution of grades among class members in time. By calculating the weighted average value of coefficient of variation increments in multiple exams, the mean of the coefficient of variation increments can be obtained, i.e

$$
\bar{\Delta}=\sum_{k=1}^{m-1} \omega_{k} \Delta_{k j}, j=1,2, \cdots, n,
$$

where $\omega_{k}, k=1,2, \cdots, m-1$ is the kth weight of the coefficient of variation increment, and the specific value can be determined according to the actual test situation.

\subsection{Rank the mean of the average score increments}

By the theory of the value-added evaluation, the mean of the average score increments which is calculated by formula (3), can eliminate the differences among different levels. Then it ranks the mean of the average score increments of each class from the largest to the smallest. The higher the mean of the average score increments is, the higher the ranking is. The smaller the mean of the average score increments is, the lower the ranking is. Thus, the class ranking $M_{j}, j=1,2, \cdots, n$, based on the mean of the average score increments is obtained, and comparison is realized which is from horizontal to vertical successfully.

\subsection{Rank the mean of the coefficient of variation increments}

By the theory of the value-added evaluation, the mean of the coefficient of variation increments which is calculated by formula (5), can further eliminate the differences among different levels. Then it ranks the mean of the coefficient of variation of each class from the smallest to the largest. The smaller the mean of the coefficient of variation is, the higher the ranking is; the higher the mean of coefficient of variation increments, the lower the ranking is. Thus, the class ranking $N_{j}, j=1,2, \cdots, n$, based on the mean of the coefficient of variation increments is obtained, and comparison is realized which is from horizontal to vertical successfully in the same way.

\subsection{Get the final ranking by value-added method}

This is correct. That the higher the class average score of a course is, the smaller the coefficient of variation is, which indicates the higher the class teaching quality is. The weighted mean of the two rankings of each class is further calculated, by comprehensively considering the mean ranking of the average score increments and the mean ranking of the coefficient of variation increments, which is denoted as,

$$
\begin{aligned}
& T_{j}, j=1,2, \cdots, n, \text { i.e } \\
& T_{j}=\alpha_{j} M_{j}+\beta_{j} N_{j} .
\end{aligned}
$$

In which, $\alpha_{j}, \beta_{j}, j=1,2, \cdots, n$ are the weights of the rank of average score increments mean and the rank of coefficient of variation increments mean respectively. The specific values can be determined according to the actual examination of the school. By sorting each class according to the order of $T_{j}, j=1,2, \cdots, n$ from small to large, the final ranking of each class is obtained by the value-added method of a course, and the teaching quality evaluation of teachers under the class-based stratified teaching mode is realized.

\section{Examples demonstration}

This paper takes the students of grade 2018 in a middle school in Qinhuangdao City as the research object, with a total of 24 classes in this grade, and the calculated results of " CEACV method" are obtained by taking three test scores of mathematics as an example.

The class rank $M_{j}, j=1,2, \cdots, 24$ is sorted from largest to smallest, based on the mean of average score increments, as shown in Table I (the data in parentheses are negative) .

Table1. The Mean Ranking of the Average Score Increments

\begin{tabular}{|c|c|c|c|c|}
\hline $\begin{array}{c}\text { The } \\
\text { classes }\end{array}$ & $\begin{array}{c}\text { The first } \\
\text { increment } \\
U_{1 j}\end{array}$ & $\begin{array}{c}\text { The second } \\
\text { increment } \\
U_{2 j}\end{array}$ & $\begin{array}{c}\text { The mean of } \\
\text { increments } \\
\bar{U}\end{array}$ & $\begin{array}{c}\text { The } \\
\text { ranking } \\
M_{j}\end{array}$ \\
\hline 21 & 7.00 & $(5.90)$ & 0.55 & 1 \\
\hline 22 & 5.04 & $(9.24)$ & $(2.10)$ & 2 \\
\hline 24 & $(1.50)$ & $(7.54)$ & $(4.52)$ & 3 \\
\hline 8 & 9.23 & $(19.91)$ & $(5.34)$ & 4 \\
\hline 10 & 10.96 & $(22.82)$ & $(5.93)$ & 5 \\
\hline 1 & 5.63 & $(17.55)$ & $(5.96)$ & 6 \\
\hline 7 & 0.57 & $(12.95)$ & $(6.19)$ & 7 \\
\hline 18 & 15.98 & $(28.78)$ & $(6.40)$ & 8 \\
\hline 2 & 6.81 & $(19.77)$ & $(6.48)$ & 9 \\
\hline 20 & 4.96 & $(18.47)$ & $(6.76)$ & 10 \\
\hline 9 & 6.91 & $(21.00)$ & $(7.04)$ & 11 \\
\hline 6 & 8.28 & $(22.63)$ & $(7.18)$ & 12 \\
\hline 4 & 6.29 & $(21.10)$ & $(7.41)$ & 13 \\
\hline 15 & 5.71 & $(21.06)$ & $(7.68)$ & 14 \\
\hline 23 & $(3.05)$ & $(12.69)$ & $(7.87)$ & 15 \\
\hline 5 & 12.04 & $(28.04)$ & $(8.00)$ & 16 \\
\hline & & & & \\
\hline
\end{tabular}




\begin{tabular}{|c|c|c|c|c|}
3 & 6.43 & $(24.04)$ & $(8.80)$ & 17 \\
\hline 19 & 3.42 & $(21.14)$ & $(8.86)$ & 18 \\
\hline 12 & 12.30 & $(30.62)$ & $(9.16)$ & 19 \\
\hline 17 & 6.11 & $(25.38)$ & $(9.64)$ & 20 \\
\hline 16 & 9.76 & $(29.33)$ & $(9.78)$ & 21 \\
\hline 11 & 7.04 & $(26.61)$ & $(9.79)$ & 22 \\
\hline 23 & $(0.00)$ & 0.07 & 0.03 & 23 \\
\hline 24 & $(0.02)$ & 0.07 & 0.02 & 24 \\
\hline
\end{tabular}

The class rank $N_{j}, j=1,2, \cdots, 24$ is sorted in order from smallest to largest, according to the mean of the coefficient of variation increments, as shown in Table II (data in parentheses are negative).

Table2. The Mean Ranking of the Coefficient of Variation Increments

\begin{tabular}{|c|c|c|c|c|}
\hline $\begin{array}{c}\text { The } \\
\text { classes }\end{array}$ & $\begin{array}{c}\text { The first } \\
\text { increment } \\
\Delta_{1 j}\end{array}$ & $\begin{array}{c}\text { The second } \\
\text { increment } \\
\Delta_{2 j}\end{array}$ & $\begin{array}{c}\text { The mean of } \\
\text { increments } \\
\bar{\Delta}\end{array}$ & $\begin{array}{c}\text { The } \\
\text { ranking } \\
N_{j}\end{array}$ \\
\hline 14 & $(0.02)$ & 0.03 & 0.00 & 1 \\
\hline 21 & $(0.01)$ & 0.05 & 0.02 & 2 \\
\hline 20 & $(0.02)$ & 0.06 & 0.02 & 3 \\
\hline 24 & $(0.02)$ & 0.07 & 0.02 & 4 \\
\hline 10 & $(0.00)$ & 0.05 & 0.03 & 5 \\
\hline 11 & $(0.02)$ & 0.07 & 0.03 & 6 \\
\hline 22 & $(0.05)$ & 0.11 & 0.03 & 7 \\
\hline 12 & $(0.01)$ & 0.07 & 0.03 & 8 \\
\hline 13 & $(0.02)$ & 0.09 & 0.03 & 9 \\
\hline 23 & $(0.00)$ & 0.07 & 0.03 & 10 \\
\hline 19 & $(0.03)$ & 0.10 & 0.03 & 11 \\
\hline 16 & $(0.05)$ & 0.14 & 0.04 & 12 \\
\hline 7 & 0.01 & 0.08 & 0.04 & 13 \\
\hline 4 & $(0.00)$ & 0.09 & 0.04 & 14 \\
\hline 18 & $(0.06)$ & 0.15 & 0.05 & 15 \\
\hline 3 & 0.02 & 0.08 & 0.05 & 16 \\
\hline 6 & 0.02 & 0.09 & 0.06 & 17 \\
\hline 8 & $(0.02)$ & 0.13 & 0.06 & 18 \\
\hline 2 & $(0.02)$ & 0.13 & 0.06 & 19 \\
\hline 17 & 0.03 & 0.09 & 0.06 & 20 \\
\hline 1 & 0.01 & 0.11 & 0.06 & 21 \\
\hline 9 & 0.03 & 0.10 & 0.07 & 22 \\
\hline 15 & 0.02 & 0.11 & 0.07 & 23 \\
\hline 5 & $(0.01)$ & 0.20 & 0.09 & 24 \\
\hline & & & & \\
\hline
\end{tabular}

According to Table I and Table II, the value-added method final ranking of mathematics of each class will be get, with using the formula (6)

$$
T_{j}=\alpha_{j} M_{j}+\beta_{j} N_{j}, j=1,2, \cdots, 24 \text { (take }
$$

$\alpha_{j}=\beta_{j}=0.5$ ), which is weighted by the mean of the average score increments and the coefficient of variation increments. Then the teaching quality evaluation of teachers is realized, as shown in Table III.

Table3. The value-added method final ranking of mathematics

\begin{tabular}{|c|c|c|c|c|}
\hline $\begin{array}{c}\text { The } \\
\text { classes }\end{array}$ & $\begin{array}{c}\text { The mean } \\
\text { ranking of the } \\
\text { average score } \\
\text { increments } \\
M_{j}\end{array}$ & $\begin{array}{c}\text { The mean } \\
\text { ranking of the } \\
\text { coefficient of } \\
\text { variation } \\
\text { increments } \\
N_{j}\end{array}$ & $\begin{array}{c}\text { Weighted } \\
\text { Overall } \\
\text { rating } \\
T_{j}\end{array}$ & $\begin{array}{c}\text { The } \\
\text { final } \\
\text { ranking }\end{array}$ \\
\hline 21 & 1 & 2 & 1.5 & 1 \\
\hline 24 & 3 & 4 & 3.5 & 2 \\
\hline 22 & 2 & 7 & 4.5 & 3 \\
\hline
\end{tabular}

\begin{tabular}{|c|c|c|c|c|}
10 & 5 & 5 & 5 & 4 \\
\hline 20 & 10 & 3 & 6.5 & 5 \\
\hline 7 & 7 & 13 & 10 & 6 \\
\hline 8 & 4 & 18 & 11 & 7 \\
\hline 18 & 8 & 15 & 11.5 & 8 \\
\hline 14 & 23 & 1 & 12 & 9 \\
\hline 23 & 15 & 10 & 12.5 & 10 \\
\hline 1 & 6 & 21 & 13.5 & 11 \\
\hline 4 & 13 & 14 & 13.5 & 12 \\
\hline 12 & 19 & 8 & 13.5 & 13 \\
\hline 2 & 9 & 19 & 14 & 14 \\
\hline 11 & 22 & 6 & 14 & 15 \\
\hline 6 & 12 & 17 & 14.5 & 16 \\
\hline 19 & 18 & 11 & 14.5 & 17 \\
\hline 3 & 17 & 16 & 16.5 & 18 \\
\hline
\end{tabular}

Continue to TABLE III

\begin{tabular}{|c|c|c|c|c|}
\hline 9 & 11 & 22 & 16.5 & 19 \\
\hline 13 & 24 & 9 & 16.5 & 20 \\
\hline 16 & 21 & 12 & 16.5 & 21 \\
\hline 15 & 14 & 23 & 18.5 & 22 \\
\hline 5 & 16 & 24 & 20 & 23 \\
\hline 17 & 20 & 20 & 20 & 24 \\
\hline
\end{tabular}

\section{Conclusion}

According to the " CEACV method", the results of this measurement were compared with the actual teaching level of teachers in each class of 2018 in the middle school, and it was found that the measurement results were in good agreement with the actual teaching level of teachers. By calculating the final ranking $T_{i}$ of teachers with "

CEACV method" in each subject, evaluation of teaching quality can be realized fairly. This evaluation method overcomes the irrationality of relying on students' absolute scores to evaluate teaching effect of teachers in the classbased stratified teaching system, and it is simple to calculate and easy to popularize. In fact, the " CEACV method" is more reasonable to evaluate the teaching quality of teachers by adding indicators, such as excellence rate and pass rate, by giving different weights to each index and then accumulating the final ranking which based on the value-added method of each class.

\section{Acknowledgments}

Social Science Development Research Project of Hebei Province in 2020: Teaching Quality Evaluation of Teachers Model Based on Student Achievements under the New College Entrance Examination Mode and It's Matlab Realization". (Item no. 20200502050)

\section{Reference}

1. Jiyu Dai. (2016) The types and characteristics of Classbased stratified teaching in our country [J]. Teaching and Management, 4: 54-56.

2. Kaijian Xiao. (2018) Suggestions on Constructing the Evaluation System of History Teaching in Hainan's High Schools with Optional Class System [D]. Hainan Normal University.

3. Yingxiao Lv. (2016) Evaluation Strategy of Teachers' 
Teaching Behavior with the Background of class selection System [D]. East China Normal University.

4. Junkao Li, Xiaoxu Hu. (2020) Reflections on the reform of teacher evaluation in senior high schools under the new college entrance examination system [J]. Academic outlook, 1: 16-19, 29.

5. Xiaomei Zhang. (2019) A brief analysis of the current relatively common several student achievement evaluation methods [J]. Students work, 1: 175-176. 